\title{
Overview of CoMP Clustering in UDN
}

\author{
Qianqian Li, Yanping Xu, Xinji Tian
}

School of Physics \& Electronic Information Engineering, Henan Polytechnic University, Jiaozuo, China

Email: tian215216@sohu.com

How to cite this paper: $\mathrm{Li}, \mathrm{Q} . \mathrm{Q}$., $\mathrm{Xu}, \mathrm{Y} . \mathrm{P}$. and Tian, X.J. (2018) Overview of CoMP Clustering in UDN. Open Access Library Journal, 5: e4703.

https://doi.org/10.4236/oalib.1104703

Received: June 1, 2018

Accepted: June 23, 2018

Published: June 26, 2018

Copyright (C) 2018 by authors and Open Access Library Inc.

This work is licensed under the Creative Commons Attribution International License (CC BY 4.0).

http://creativecommons.org/licenses/by/4.0/

\section{(c) (†) Open Access}

\begin{abstract}
System throughput and resource utilization can be improved in ultra-dense networks (UDN) by deploying a large number of small base stations. However, base stations are very close in UDN, which will cause serious interference between base stations. To solve this problem, coordinated multi-point (CoMP) has been improved, which has the ability to reduce inter-cell interference and to improve data rate. However, the complexity of cooperation among all cells is very high. Therefore, it is necessary to construct small-size cooperation clusters. This article analyzes one of the key challenges of CoMP application in UDN-optimal CoMP clustering. Firstly, the background knowledge of CoMP is introduced. Secondly, we focus on the classification and summarization of the latest achievements in clustering research and the objective and challenges faced in the design of CoMP clusters. Finally, the future research directions and potential methods of CoMP clustering are discussed.
\end{abstract}

\section{Subject Areas}

Mobile and Portable Communications Systems

\section{Keywords}

Ultra-Dense Networks, Coordinated Multi-Point, Complexity, CoMP

Clustering

\section{Introduction}

With the growth of economy and the development of science and technology, people's living standards have been continuously improved. The demand for communications has grown from the initial voice communication to today's data, streaming media and other real-time services. The application scenario involves the lives and work of people. With the popularity of various new mobile terminals such as smart phones and tablet computers, mobile data traffic has 
grown exponentially. Cisco predicts that the demand of wireless data traffic will explode. In 2020, the 1000-fold increase in mobile data traffic is expected for $5 \mathrm{G}$ beyond 2020 [1]. To cope with the tremendous growth of wireless traffic in $5 \mathrm{G}$, three development directions are proposed in [2] [3]: 1) UDN: massive small cell deployment; 2) increase spectrum efficiency-coordinated multiple points (CoMP), multiple input multiple output (MIMO), and enhanced coding techniques; 3 ) add additional spectrum. Network density can increase the capacity of the system by 40 - 50 times. The system capacity can be increased by 3 - 5 times through increasing the spectrum efficiency. The system capacity can be increased by 5 - 10 times through increasing additional spectrum efficiency. The UDN can provide the greatest network capacity gain, but it needs to deploy a large number of small base stations [4].

In the world's major operator data services, $60 \%$ of mobile voice services and $90 \%$ of data services occur indoor and hot spots. Therefore, solving the problem of rapid increase of indoor coverage and hot spot business is an important way to improve system capacity. Heterogeneous wireless networks incorporating a variety of wireless access technologies are more capable of meeting the potential requirements of wireless communications. With the rapid increase in the number and density of users, UDNs with densely deployed nodes have become the future trend of wireless communications [5]. The small cell can reuse the frequency of the macro cell. The small base station has a low transmission power and a small coverage area. It can be deployed in a large number in the networks, forming a high frequency reuse rate. However, there are still many technical challenges to be resolved if excellent performance of UDN is to be achieved. The small station reuses the frequency band of macro cell in UDN, which brings about severe co-channel interference between the macro cell user and the small cell or co-channel interference between the small cell users. Interference becomes a major obstacle of UDN development.

Traditional interference management strategies, such as power control and channel quality-based scheduling, can suppress intra-cell interference to a certain extent. However, it performs interference control based on user information in a single cell, and does not interact with neighboring cells. The ability to handle inter-cell interference is very limited [6]. Therefore, CoMP has been proposed to reduce inter-cell interference and to improve high data rate coverage and cell edge throughput. CoMP has been introduced for long term evolution advanced (LTE-A) by the third generation partnership project (3GPP) in [7]. However, the application of this technique requires precise synchronization requirement, additional pilot spending and signal and scheduling management among all base stations. Therefore, the complexity of collaboration between all cells in the network is extremely high [8]. To reduce complexity, it is necessary to build a small-size cooperation cluster, in which the base stations cooperate with each other. Designing the optimal CoMP cluster is one of the key challenges for CoMP implementation for future wireless networks [9]. 


\section{UDN Overview}

The UDN is composed of a large number of small cells. The small cell, as low-power wireless access node, not only can work on licensed frequency bands, but also can work on unlicensed frequency bands. The coverage of macro base station is several kilometers, while that of small cells is generally between 10 and 200 meters. Microcells, picocells, and femtocells can all be considered as one type of small cell. The microcells with transmission power between $5 \mathrm{~W}$ and 10 $\mathrm{W}$, are used for outdoor coverage. Its coverage radius is up to 300 .

Compared to microcells, picocells have relatively low power, and its coverage radius is generally between 50 meters and 100 meters. It is mainly for some public hot spots and for outdoor coverage. Unlike the previous two types, femtocells are mainly used for indoor coverage. Its transmission power is generally within 100 milliwatts, and its coverage is between 10 and 20 meters [10].

You can apply small cells in the following scenarios:

1) Coverage hole. Due to terrain restrictions or building blockage, there will be some holes in the coverage of macro base stations. If new macro base stations are deployed in these holes, which can increase the overhead of network deployment. However, it would cause severe interference to the existing base stations. Covering the holes of the network through small cells can solve these problems very well. Firstly, the overhead is low, since the micro base station is cheap and easy to deploy. Secondly, the interference to the existing base station is smaller since the micro base station has lower transmission power.

2) Hot spots. In some densely populated hotspots, such as movie theaters, gymnasiums, and shopping malls, macro base stations will not be able to provide users with satisfactory services due to resource constraints. In this case, service splitting can be implemented by deploying small cells, thereby reducing the transmission load of the macro base station.

\section{CoMP}

CoMP is one of the main methods to mitigate the average interference and to increase the spectral efficiency [11]. CoMP aims to transmit data for users and to enhance the user's service experience by the cooperation between multiple transmission points in different cells. It has the ability to reduce inter-cell interference through information exchange between cooperative points, thereby improving user service quality [12]. CoMP technology enables users to receive useful information from multiple cells on the same time-frequency resources, thereby transforming the interference signals into useful signals, improving cell-edge spectrum efficiency, and expanding high rate data service coverage [13]. The multiple transmission points in the CoMP do not only refer to the cooperation among the evolved Node Base stations (eNBs) but also refer to the radio frequency units. Due to the development of heterogeneous networks in LTE-A networks, this collaboration based on lower levels will become the mainstream [14]. 
Inter-cell interference can be reduced by cell cooperation. By sharing the data between the coordinated points, interference can be mitigated at the receiving terminal or can be converted into the useful signal. The 3GPP further studied the CoMP in LTE-A, and also studied the concept of the physical layer of CoMP. The research results show that the CoMP can provide great performance improvement for different network deployment scenarios, especially for the cell-edge users [15].

Three main downlink coordination schemes are identified by 3GPP for LTE-Advanced.

1) Joint Transmission (JT): In CoMP-JT, users receive downlink information from multiple transmission nodes at a time and combine the information to improve received signal quality [16]. Cooperative nodes can convert the interference signal into useful signals by sharing user information [17]. For edge users, using JT can effectively improve the user's spectrum efficiency and throughput. However, JT requires the sharing of channel state information (CSI) and user data among transmission nodes, which requires high bandwidth backhaul links [18].

2) Dynamic point selection (DPS): The system provides data services for the user to select the transmission point with the best channel conditions [19]. In the DPS transmission mode, only one transmission point transmits data for the user at a time, and other transmission points do not perform data transmission for the user, but the transmission point of the transmission data varies with the time slot among the cooperation transmission points. System always selects the best transmission point to transmit data for the user [20].

3) Coordinated scheduling/beamforming (CS/CB): CS/CB is another implementation of CoMP. Unlike JT, in the CB/CS implementation process, the user's control signals and data signals are completely provided by a node, i.e., the user's serving nodes, but the user's scheduling decision and pre-coding scheme are jointly determined by multiple nodes [21]. Therefore, CS/CB is a kind of interference avoidance technology. Compared with JT, CS/CB lacks the diversity gain that JT can bring. However, the CS/CB only needs to share CSI among the cooperating nodes. Therefore, it requires low bandwidth backhaul links [22]. Coordinated scheduling means that through multiple cell collaborations, only one base station provides services for users as far as possible, a reasonable and efficient allocation of wireless resources is performed, and resources of sector boundaries are reduced in time domain, frequency domain, and airspace. So, inter-cell interference can be avoided as much as possible, and maintaining high spectrum efficiency and improving the user's receiving signal to interference plus noise ratio (SINR). The goal of CB is to form the best combination or allocation of baseband signals based on system performance indicators. Specifically, its main task is to compensate for signal fading and distortion caused by factors such as space loss and multipath effects in the wireless propagation process, and to reduce the user's co-channel interference [23]. 
Two main uplink CoMP categories are identified by 3GPP for LTE-Advanced in [24].

1) Coordinated scheduling/beamforming $(\mathrm{CS} / \mathrm{CB})$ : The cooperating transmission nodes jointly design the user's scheduling and precoding, and only one transmission node receives user data [25].

2) Joint reception: The user's data is received by multiple transmission nodes. Similar to the downlink JT, the joint reception of the uplink can provide high gains, but at the expense of increased complexity and high bandwidth backhaul links.

\section{CoMP Clustering}

At present, many domestic and foreign literatures have studied the CoMP clustering algorithm, among which there are three main clustering modes, static clustering, semi-dynamic clustering and dynamic clustering.

The static clustering method is less complex and has less signaling overhead, but this method dynamically adapts to changes in the location of network nodes or users. Therefore, its performance is limited [26]. Semi-dynamic clustering is proposed on the basis of static clustering. Static clustering is performed first, and then base stations or users are dynamically selected within the cluster. Compared with static clustering, semi-dynamic clustering improves the performance of the system. Its disadvantages are increased complexity and signaling overhead. In order to adapt to the dynamic changes of network and user location, dynamic clustering is proposed. The performance of this method is optimal, but its complexity is the highest [27]. Dynamic clustering is divided into three categories: network-centric clustering, user-centric clustering, and hybrid clustering. In the network-centric clustering method, the serving base station of the user of the same cluster can only be selected within the cluster [28]. In the user-centric clustering method, users can select their own serving base station in the whole networks, but its complexity will increase. The hybrid method is a combination of the first two methods and can be a compromise between complexity and performance [29].

\subsection{Static Clustering}

The static clustering method pre-designs a fixed cooperative cluster that does not change with time according to the radio access network structure and the base station geographic location. The advantage is that the operation is simple and complexity is extremely low; the disadvantage is that the time-varying characteristics of the fading channel are not considered and the available cooperative gain is limited.

A static clustering scheme is proposed in [30], which can improve SINR of users. In [31], static CoMP clustering is considered with orthogonal frequency reuse where antenna bore-sights are shifted to face into each other for extra CoMP gain. However, both papers assume that the cells are hexagonal grid 
which is non-realistic in real network deployment, and this topology creates blind spots. Overlapping and non-overlapping static clustering schemes are proposed in [32] where static clusters are formed to maximize the average SINR or minimizing the SINR of users. In an overlapping clustering scheme, a cell can locate three clusters simultaneously. This scheme can be applied to the real network. However, its complexity will increase as the number of users increases [33].

Static clustering is an attractive method because of its low complexity. The use of CoMP technology between base stations does not require high-bandwidth backhaul links [34]. However, static clustering algorithms are mostly based on hexagonal networks, which is unrealistic. Therefore, this method cannot provide spectral efficiency gains and increase system capacity for future $5 \mathrm{G}$ networks [35].

\subsection{Semi-Dynamic Clustering}

In order to improve performance of the network, semi-dynamic clustering is proposed on the basis of static clustering, which is a multi-layer static cluster. In semi-dynamic clustering, users select an optimal cluster from static clusters. Based on the hexagonal topological network, a clustering algorithm is proposed in [36] where it expands the two-layer static clustering into multi-layer dynamic clustering. The user selects the most suitable cluster from the available clusters based on power. This algorithm improves the cooperative gain compared with static clustering. However, its overlapping property increases the complexity of the scheduling and the bandwidth of the backhaul link. A semi-dynamic clustering method based on a hexagonal network is proposed in [37]. By assigning different sub-channels to each moving cluster, multiple clusters can be transferred. The statically clustering algorithm is studied in [38] for maximizing the number of neighboring cells in the same cluster, which proposes a complete transfer based on the static transfer in [37]. The algorithm can reduce the inter-cluster Interference and increase CoMP gain by allocates different spectrums to the transferred clusters. However, these methods are based on the hexagonal topology network and cannot be applied in actual networks. For the downlink time-division duplex JT-CoMP system, a semi-dynamic clustering method is proposed in [39]. This method divides large-size static clusters into different sub-clusters. The user dynamically selects sub-cluster. With the same spectral efficiency, its complexity is reduced. However, this method cannot respond to dynamic changes within static clusters.

Semi-dynamic clustering is an improved method of static clustering. However, most clustering algorithms are based on an ideal hexagonal network topology, which is unrealistic in practical networks [40]. In addition, most semi-dynamic clustering algorithms design static clusters by assigning orthogonal frequencies to each cell, which reduces the spectral efficiency of the network, and the static nature of clusters cannot respond to changes in users and networks [41]. 


\subsection{Static Clustering}

Dynamic clustering is designed periodically according to the change of fading channel information (usually considered to be clustering with time slots as periods), can adapt to network changes and can provide higher cooperative gain. But this type of clustering operation relatively complex, periodic clustering makes the computational complexity increase exponentially [42] [43]. Dynamic clustering is divided into three categories: network-centric clustering, user-centric clustering, and hybrid clustering [44].

Network-centric clustering methods are less complex when compared to user-centric clustering [45], but cluster edge users still suffer from inter-cluster interference [46]. Network-centric clustering methods mostly use greedy algorithms and cooperative game theory. In [47] aiming at maximizing spectral efficiency, a greedy clustering algorithm for uplink is proposed. The cluster size is set in advance. This algorithm indicates that dynamic clustering with cluster size of two cells outperforms static clustering with larger cluster size. However, the size of the clusters fixed in advance is not optimal solution for some clusters. An improved greedy clustering algorithm is proposed in [48]. The size of the cluster is not fixed, but varies with the CSI. Both [48] and [49] are non-overlapping clusters and the inter-cluster interference is still high. A dynamic overlapping clustering method based on greedy algorithm is proposed in [50], which can improve the network average rate and fairness. This paper also showed that the performance of four-size overlapping clusters is better than that of eight-size non-overlapping clusters. Cooperative game theory can also be used for clustering. Using the utility function in cooperative game theory, the size of the cluster can be limited according to the location of the base station and the user configuration, so that the CoMP gain can be maximized. A continuous second-order cone programming method is proposes in [51] to solve the joint processing and clustering problems, which can greatly reduce the scope of the cluster and can reduce the backhaul load.

The user-centric dynamic clustering selects each user's serving base station cluster among all small base stations according to the user's CSI. This method can obtain better SINR and throughput gain with the highest complexity [52]. In order to reduce the complexity, the user-centric clustering method cannot be applied to the entire network and can only be used in an area containing several serving base stations [53]. The research in [54] shows that using macro-diversity CoMP in user-centric dynamic clustering can improve system capacity, and [55] proposes a three-tier clustering method, in which cell center users do not use CoMP technology and perform static clustering. User-centric dynamic clustering is used for cluster edge users. The complexity of the methods proposed in [54] and [55] is similar, where does not limit the number of user-centric cells. When the number of cells is large, the complexity of these two methods is extremely high. In [56], a user-centric CoMP clustering algorithm is studied under the premise of a certain range of clusters, which can maximize spectrum effi- 
ciency and introduce a new two-stage re-clustering algorithm for multi-user collaboration. In the case of CoMP transmission, the load is transferred from the high-loaded cell to the low-loaded cell to reduce the high load in the hot spot and improve the user satisfaction. In [57], a user-centric overlapping clustering based on the main base station pre-coding is proposed for maximizing the system throughput. This method can dynamically configure the serving base station cluster in a distributed manner. However, the complexity of the algorithm is extremely high. In order to reduce the complexity, a suboptimal clustering algorithm that does not consider the interference is proposed.

Hybrid clustering is a combination of network-centric clustering and user-centric clustering methods. Each user has several candidate serving base stations, and selects serving base station clusters for each user from alternative serving base stations, and can make adjustments based on changes in the CSI [58]. Hybrid clustering can achieve a trade-off between complexity and cooperative gains [59]. A hybrid clustering method is proposed in [60] where cell-center users use network-centric clustering methods, and cell-edge users use user-centric clustering methods, to maximize the SINR of users at the cell edge. Each cell edge user selects a cluster of serving base stations and eliminates the inter-cluster interference of overlapping clusters by allocating orthogonal frequencies for different users' serving base station clusters. Although this method improves the performance of cluster edge users, the allocation of orthogonal frequencies affects the reasonable use of bandwidth. In [61], hybrid clusters in the downlink is studied, where firstly clustered the network statically according to network-centric clustering methods, and then the users in the static cluster used the user-centric clustering to select three services base station. Although the complexity of the method is low, the need to introduce dynamic clustering and to select a fixed number of serving base stations for each user will lead to unnecessary cooperation of base stations and waste of resources.

\section{The Objective of CoMP}

The main objective of CoMP is to mitigate inter-cell interference and improve spectrum efficiency, but the CoMP clustering algorithm needs to consider multiple indicators. The implementation of CoMP is limited by the bandwidth of the backhaul link. As the mobile data grows exponentially, the design of the CoMP cluster needs to take into account system capacity and load balancing. The green communication required for the fifth generation communication system should also be considered in the CoMP clustering algorithm. Next we describe in detail the objective factors that need to be considered in the design of CoMP cluster.

\subsection{Spectral Efficiency}

Wireless spectrum resource is one of the most important resources for wireless communication. The shortage of spectrum resources has become the bottleneck of the rapid development of current wireless communication technologies. The 
traditional fixed spectrum allocation method leads to the low utilization of spectrum resources. Under the condition that the overall spectrum resources are limited, under the condition of limited spectrum resources, improving spectrum resource utilization is the only way to solve the shortage of spectrum resources.

The main objective of CoMP is to reduce the inter-cell interference, improving the user's receiving SINR and the spectrum efficiency. Therefore, maximizing spectral efficiency has become the main consideration for CoMP cluster design. The distributed interference management is studied in [62] in a multi-cell multiple-input multiple-output time-division duplex network downlink. User-centric clusters are built, where users are connected to multiple base stations. These base stations serve users through CoMP-JT. The goal of this paper is to allocate appropriate serving base stations and precoding matrices for each user. Then, the interference and base station transmit power are controlled to maximize the system's spectral efficiency. Because the same frequency spectrum is reused among the same user clusters in the UDN, there may be strong interference between them. For the downlink transmission of UDN in [63], an interference graph is first created based on large-scale fading according to the interference strength between user clusters. Then, the user clusters with strong interference are merged to form a new user cluster, and the intra-cluster interference is eliminated through the CoMP-JT, which can improve the average spectrum efficiency of the user, especially the spectrum efficiency of the edge user. However, the small base stations use same power to transmit signals for users in the method, and power allocation and resource scheduling are not considered. A resource allocation scheme based on interference coordination is proposed in [64] for solving the inter-cell interference problem in UDN. First, small cells with strong interference are grouped into the same cluster based on the interference strength among the small cells, and the small cells in the same cluster share frequency band resources. Then based on the max-min fairness criteria in power, optimal power allocation is performed and resources are dynamically allocated. In order to mitigate the severe interference among dense small cells and improve the performance of cell edge users, hybrid clustering based on non-cooperative game theory is proposed in [65], where SINR measurement mechanism are introduced, Non-cooperative game is used to help users select the best serving cell through considering the load of the cell, so as to achieve the purpose of improving spectrum efficiency. For CoMP can only eliminate intra-cluster interference but cannot mitigate inter-cluster interference, a dynamic joint processing scheme is proposed in [66]. Through the joint consideration of network-centric clustering methods and sub-band allocation, a single sub-band cluster is constructed. It can eliminate users at the edge of the cluster, which can reduce inter-cluster interference and improve system spectral efficiency.

Dynamic clustering of UDNs requires complex precoding design and resource allocation, and additional the overhead on the backhaul link. Semi-dynamic clustering and static clustering can reduce complexity and extra overhead at the 
expense of reducing spectral efficiency gains.

\subsection{Energy Efficiency}

UDN is one of the most advanced technologies to deal with the increasing traffic demand of wireless communications, which provide high-speed data transmission by deploying dense small base stations. However, a large number of small base stations have brought a lot of energy consumption and maintenance costs. The increasingly serious greenhouse effect has also become an important threat to the sustainable development of human beings in the future. Green communication has become the pursuit of communication development. Therefore, with the increase of spectral efficiency, energy efficiency has also become a major concern of UDN. At present, for the research of green communication, common methods are base station sleeping and CoMP technology.

According to IEEE 802.16 standard, even if the small base station works in low load mode, the base station still has a certain amount of time in the detection state and consumes energy without any service. In order to save this part of energy, the topology structure of the network is known information in [67], the small base stations are clustered and the cluster heads are selected based on their geographic location. In the low-load operation mode, only the cluster heads need to perform detection intermittently for new user, so that users entering the service area can know the which cluster they am in. When the cluster head discovers a new user, it determines which base station the user belongs to according to the location of the user and wakes up the corresponding small base station. After improvement, when there are few services, only the cluster head needs to work, while other members save the energy consumed by periodic detection.

The network densification will greatly increase energy consumption and operating costs. Because CoMP technology can reduce the transmission power of small base stations, it can improve energy efficiency. To maximize energy efficiency, and optimize the trade-off between spectrum efficiency and energy efficiency, has been concentrated on the important factors for design of CoMP clusters. However, extra signal processing and backhaul links of CoMP also consume energy. When the density of active small base stations is different, the performance of the network is analyzed in [68]. Finally, according to the different performance indicators of the network, trade-off between the spectrum efficiency and energy efficiency are taken into account by changing the density of active small base stations.

Energy saving base station is an important design goal in a green cellular network. One effective way to save energy at the base station is to turn the base station on or off based on the network traffic conditions. However, this method may cause network coverage holes. In [69], a method that adaptively switching the base station on or off to minimize the total power consumption of the network is studied while maintaining network coverage, which has been proved to save network energy in the hot-spot area where dense small base stations are deployed. CoMP clusters can optimize network energy efficiency by increasing 
the number of small base stations that work in sleep mode or increasing the sleep time of small cells. Downlink CoMP transmission links is considered in [70] to minimize the total power consumption of the network while satisfying the user's requirements of the SINR and base station power constraints through jointly optimize the base station mode (active or sleep), user connection methods and cooperative beamforming. In addition to letting the small base station sleep, considering network coordination in the network planning stage can also reduce deployment costs and maximize energy efficiency.

\subsection{Backhaul Optimization}

Most of the existing research only considers the link between the user equipment and the base station, and does not consider the backhaul link between the base station and the core network. The backhaul link may be wired or wireless, but its capacity is limited, therefore there is a delay in the backhaul link, which can affect real-time nature of information exchange between cells, so that each cell cannot get the decisions and information about neighboring cells in time. Incomplete or inaccurate acquisition of network information by the base station will cause decision errors and degrade system performance. The delay of information means that, the base station or user can only make decisions based on historical information and cannot adapt to the current network conditions in time. The condition of the network has seriously affected the performance of the system. As mentioned earlier, one of the prerequisites for CoMP applications is high backhaul bandwidth and low latency. Due to the need to share user data and CSI among cells in a cluster, JT-CoMP requires a high backhaul bandwidth.

A joint optimized beamforming design and user-centric clustering is proposed in [71] for the large backhaul signaling overhead caused by CoMP. The method clusters in a given network-centric cluster to reduce wasted network resources. A semi-distributed algorithm was proposed to solve this problem and its suboptimal solution was obtained. Simulation results show that the proposed method can reduce backhaul data transmission by $10 \%-30 \%$. In [72], in the precoder design, the long-term channel statistics composed of path loss and shadow fading are used to simulate the unknown CSI interference, which can reduce the burden on the backhaul link. In order to maximize the weighted sum rate, a continuous second-order cone algorithm is proposed, which is solved by the branch-and-bound method. An alternative optimization scheme for minimizing the weighted mean square error has also been derived. These two schemes provide a near-optimal effective solution and enable efficient transmission of backhaul links.

CoMP implementation relies on high backhaul bandwidth, so the CoMP clustering algorithm needs to consider backhaul bandwidth limitations. Cluster size and collaboration type are also important factors that can change the backhaul bandwidth requirements. In addition, in order to obtain better CoMP gains, the data of the high load backhaul link can be transferred to the backhaul link of the low load cell. 


\section{Challenges and Development Directions}

\subsection{The Trade-Off between Complexity and Gain}

Because dynamic clustering can respond to the dynamic changes of base stations and users in time, UDN can achieve better performance gains. Inter-cluster interference can be mitigated by dynamically changing cluster boundaries. The user-centric, network-centric, and hybrid clustering methods have been analyzed above. The study of CoMP clusters for user-centric approaches lacks scalability and the complexity of scheduling and precoding designs is extremely high. Compared with the user-centric cluster design, the network-centric approach cannot provide the full benefits of CoMP. Hybrid clustering can make a trade-off between the complexity and gains of CoMP clustering. However, the existing research work cannot be completely dynamic hybrid clustering scheme. Because the complete dynamic clustering scheme is too complicated and has additional overhead.

Therefore, the selection of the clustering method must rely on the practical networks environment and user service quality requirements. Under meeting the basic service requirements of users, the complexity is too high to be worth the loss. So the complexity of the clustering method should be reduced as much as possible. Clustering methods have no advantages and disadvantages, depending on whether they can meet the actual needs of the network.

\subsection{CoMP Clustering for Multiple Objectives}

Nowadays, the objects of Operators tend to be diversified and face various business demands. Users and services are characterized by diversification and heterogeneity. Various Operators providers must ensure that they can provide high quality services in order to gain an advantage in the fierce market competition.

However, CoMP is mainly used to mitigate inter-cell interference and increase spectrum efficiency. Therefore, the main objective of CoMP clusters is to increase spectrum efficiency, but there are other limitations such as backhaul bandwidth, system capacity and energy efficiency. Most of the papers are concerned with two aspects of research. One is to maximize spectral efficiency and the other is to focus on the limits of backhaul bandwidth and energy efficiency. In order to meet the high quality service requirements of each user as much as possible, it is necessary to more fully consider all the constraints to achieve the CoMP clusters design. It is necessary to research on clustering algorithms that comprehensively consider various constraints, and incorporate various objectives, i.e., spectrum efficiency, energy efficiency, backhaul bandwidth, and load into algorithm design, while considering a trade-off between multiple objective gains and additional overhead. Tools such as joint game theory can combine multiple objectives into a single benefit function. The trade-off between different objectives and the best balance between these objectives is a field worthy of study. 


\section{Conclusion}

This paper mainly analyzes UDN and CoMP clusters extensively. First of all, it analyzes the reason why UDN becomes the key technology of the future 5G network, as well as its advantages and disadvantages. Then the classification of various CoMP clusters is introduced, which analyzes the advantages and disadvantages of various clustering methods. Moreover, a new classification method of CoMP cluster based on objective function is proposed. The CoMP clustering algorithm optimized for spectrum efficiency, energy efficiency and backhaul is discussed. Finally, the challenges and development directions of CoMP clusters are discussed in the future.

\section{References}

[1] (2016) Cisco Visual Networking Index: Global Mobile Data Traffic Forecast Update, 2015-2020. White Paper, Cisco, San Jose, CA, USA.

[2] Li, Q.C., Niu, H., Papathanassiou, A.T., et al. (2014) 5G Network Capacity: Key Elements and Technologies. IEEE Vehicular Technology Magazine, 9, 71-78. https://doi.org/10.1109/MVT.2013.2295070

[3] Imran, A. and Zoha, A. (2014) Challenges in 5G: How to Empower SON with Big Data for Enabling 5G. Network IEEE, 28, 27-33. https://doi.org/10.1109/MNET.2014.6963801

[4] Jungnickel, M.K., Zirwas, W., et al. (2014) The Role of Small Cells, Coordinated Multipoint, and Massive MIMO in 5G. IEEE Communications Magazine, 52, 44-51. https://doi.org/10.1109/MCOM.2014.6815892

[5] Wang, Y., Miao, Z.Y. and Zhang, P. (2015) Research Progress and Key Technologies in Ultra Dense Networks. Journal of Beijing University of Posts and Telecommunications, 38, 1-17.

[6] Balachandran, K., Kang, J.H., Karakayali, K., et al. (2011) NICE: A Network Interference Cancellation Engine for Opportunistic Uplink Cooperation in Wireless Networks. IEEE Transactions on Wireless Communications, 10, 540-549. https://doi.org/10.1109/TWC.2010.120610.100169

[7] Cisco Incoporated. (2016) Cisco Visual Networking Index: Global Mobile Data Traffic Forecast Update, 2015-2020. White Paper, CA, USA.

[8] Irmer, R., Droste, H., Marsch, P., et al. (2011) Coordinated Multipoint: Concepts, Performance, and Field Trial Results. IEEE Communications Magazine, 49, 102-111. https://doi.org/10.1109/MCOM.2011.5706317

[9] Bassoy, S., Farooq, H., Imran, M.A., et al. (2017) Coordinated Multi-Point Clustering Schemes: A Survey. IEEE Communications Surveys \& Tutorials, PP, 748-749.

[10] Han, S.Q., Yang, C.Y., Li, G.Y., et al. (2017) Energy Efficient Resource Optimization and Transmission. Scientia Sinica Informationis, No. 5.

[11] Mosleh, S., Liu, L. and Zhang, J. (2016) Proportional-Fair Resource Allocation for Coordinated Multi-Point Transmission in LTE-Advanced. IEEE Transactions on Wireless Communications, 15, 5355-5367. https://doi.org/10.1109/TWC.2016.2557328

[12] Zhu, L., Yu, F.R., Wang, H., et al. (2015) A Novel Communication-Based Train Control (CBTC) System with Coordinated Multi-Point Transmission and Reception. IEEE Global Communications Conference, Austin, TX, 8-12 December 2014, 
$4377-4381$.

[13] Jia, H.Y. (2011) Research of Coordinated Multiple Point Transmission Technology in LTE-A Based on System Level Simulation. Beijing Jiaotong University, Beijing.

[14] Chen, G. (2015) Research on CoMP-JP Technology. Beijing University of Posts and Telecommunications, Beijing.

[15] Huang, Z.J. (2015) Research on the Coordinated Multi-Point Transmission Technology. Beijing University of Posts and Telecommunications, Beijing.

[16] Chen, P.C. (2017) Load-Aware Coordinated Multi-Point Joint Transmission in Dense Heterogeneous Networks.

[17] Zuo, W., Xia, H.L. and Feng, C. (2015) A Novel Coordinated Multi-Point Transmission in Dense Small Cell Deployment. International Symposium on Personal, Indoor, and Mobile Radio Communications, Hong Kong, 30 August-2 September 2015, 1872-1877.

[18] Beylerian, A. and Ohtsuki, T. (2016) Multi-Point Fairness in Resource Allocation for C-RAN Downlink CoMP Transmission. EURASIP Journal on Wireless Communications \& Networking, 2016, 12. https://doi.org/10.1186/s13638-015-0501-4

[19] Bradai, A., Benslimane, A. and Singh, K.D. (2015) Dynamic Anchor Points Selection for Mobility Management in Software Defined Networks. Journal of Network and Computer Applications, 57, 1-11. https://doi.org/10.1016/j.jnca.2015.06.018

[20] Apilo, O., Lasanen, M. and Mämmelä, A. (2016) Energy-Efficient Dynamic Point Selection and Scheduling Method for Intra-Cell CoMP in LTE-A. Wireless Personal Communications, 86, 705-726. https://doi.org/10.1007/s11277-015-2953-6

[21] Ha, V.N. and Long, B.L. (2015) Joint Coordinated Beamforming and Admission Control for Fronthaul Constrained Cloud-RANs. Global Communications Conference, San Diego, 6-10 December 2015, 4054-4059.

[22] Huang, X., Xue, G., Yu, R., et al. (2016) Joint Scheduling and Beamforming Coordination in Cloud Radio Access Networks with QoS Guarantees. IEEE Transactions on Vehicular Technology, 65, 5449-5460.

https://doi.org/10.1109/TVT.2015.2464278

[23] Gao, W. and Cui, Q. (2015) Adaptive Coordinated Scheduling/Beamforming Scheme for Downlink LTE-Advanced System with Non-Ideal Backhaul. International Symposium on Wireless Personal Multimedia Communications, Hyderābād, 13-16 December 2015, 356-361.

[24] Peng, H., Xiao, Y., Ruyue, Y.N., et al. (2016) Ultra Dense Network: Challenges, Enabling Technologies and New Trends. China Communication, 13, 30-40.

[25] Shen, K. and Yu, W. (2016) Coordinated Uplink Scheduling and Beamforming for Wireless Cellular Networks via Sum-of-Ratio Programming and Matching. IEEE International Conference on Acoustics, Speech and Signal Processing, Shanghai, 20-25 March 2016, 3531-3535.

[26] Zeng, X., Sun, M., Jian, X., et al. (2017) Optimal Base Stations Planning for Coordinated Multi-Point System. International Journal of Electronics and Communications, 73, 193-201. https://doi.org/10.1016/j.aeue.2017.01.007

[27] Kang, H.S. and Kim, D.K. (2016) User-Centric Overlapped Clustering Based on Anchor-Based Precoding in Cellular Networks. IEEE Communications Letters, 20, 542-545. https://doi.org/10.1109/LCOMM.2016.2515085

[28] Li, C. (2016) The Research on the Cooperating Nodes Selection in Co MP of LTE-Advanced System. Huazhong University of Science and Technology, Wuhan.

[29] Li, S., Zhu, G., Lin, S., et al. (2017) Cross-Layer Resource Management in Software 
Defined Ultra Dense Wireless Networks. International Conference on Mobile Ad-Hoc and Sensor Networks, Beijing, 17-20 December 2017, 412-416.

[30] Ali, S.S. and Saxena, N. (2013) A Novel Static Clustering Approach for CoMP. International Conference on Computing and Convergence Technology, Seoul, 3-5 December 2012, 757-762.

[31] Shimodaira, H., Tran, G.K., Araki, K., et al. (2016) Diamond Cellular Network-Optimal Combination of Small Power Basestations and CoMP Cellular Networks. International Symposium on Personal, Indoor and Mobile Radio Communications, Valencia, 4-8 September 2016, 163-167.

[32] Marsch, P. and Fettweis, G. (2011) Static Clustering for Cooperative Multi-Point (CoMP) in Mobile Communications. IEEE International Conference on Communications, Kyoto, 5-9 June 2011, 1-6.

[33] Yang, S., Wang, W., Liu, C., et al. (2015) Optimal Reactive Power Dispatch of Wind Power Plant Cluster Considering Static Voltage Stability for Low-Carbon Power System. Journal of Modern Power Systems \& Clean Energy, 3, 114-122. https://doi.org/10.1007/s40565-014-0091-x

[34] Wahdan, M.A., Al-Mistarihi, M.F. and Shurman, M. (2015) Static Cluster and Dynamic Cluster Head (SCDCH) Adaptive Prediction-Based Algorithm for Target Tracking in Wireless Sensor Networks. International Convention on Information and Communication Technology, Electronics and Microelectronics, Opatija, 25-29 May 2015, 596-600. https://doi.org/10.1109/MIPRO.2015.7160342

[35] Cerezo, J., Petrone, A., Ferrer, F.J.A., et al. (2016) Electronic Spectroscopy of a Solvatochromic Dye in Water: Comparison of Static Cluster/Implicit and Dynamical/Explicit Solvent Models on Structures and Energies. Theoretical Chemistry Accounts, 135, 263. https://doi.org/10.1007/s00214-016-2009-3

[36] Huang, F., Wang, Y., Geng, J., et al. (2010) Clustering Approach in Coordinated Multi-Point Transmission/Reception System. Vehicular Technology Conference, Taipei, 16-19 May 2010, 1-5.

[37] Ramprashad, S.A., Caire, G. and Papadopoulos, H.C. (2010) A Joint Scheduling and Cell Clustering Scheme for MU-MIMO Downlink with Limited Coordination. IEEE International Conference on Communications, Cape Town, 23-27 May 2010, 1-6.

[38] Pichapati, V. and Gupta, P. (2013) Practical Considerations in Cluster Design for Coordinated Multipoint (CoMP) Systems. IEEE International Conference on Communications (ICC), Budapest, 9-13 June 2013, 5860-5865.

[39] Sun, H., Zhang, X. and Fang, W. (2012) Dynamic Cell Clustering Design for Realistic Coordinated Multipoint Downlink Transmission. International Symposium on Personal Indoor and Mobile Radio Communications, Sydney, 9-12 September 2012, 1331-1335.

[40] Zhang, H., Liu, H., Jiang, C., et al. (2015) A Practical Semidynamic Clustering Scheme Using Affinity Propagation in Cooperative Picocells. IEEE Transactions on Vehicular Technology, 64, 4372-4377. https://doi.org/10.1109/TVT.2014.2361931

[41] Liu, D., Han, S., Yang, C., et al. (2016) Semi-Dynamic User-Specific Clustering for Downlink Cloud Radio Access Network. IEEE Transactions on Vehicular Technology, 65, 2063-2077. https://doi.org/10.1109/TVT.2015.2431917

[42] Baracca, P., Boccardi, F. and Benvenuto, N. (2014) A Dynamic Clustering Algorithm for Downlink CoMP Systems with Multiple Antenna UEs. EURASIP Journal on Wireless Communications \& Networking, 2014, 125. https://doi.org/10.1186/1687-1499-2014-125

[43] Baracca, P. and Aziz, D. (2014) Clustering and Precoding Design for CoMP-CB in 
Downlink Heterogeneous Networks. International Symposium on Wireless Communications Systems, Barcelona, 26-29 August 2014, 59-63.

[44] Mao, Y. and Li, V.O.K. (2016) Cluster-Based Resource Allocation with Adaptive CoMP in Multi-Cell MU-MIMO OFDMA System. IEEE International Conference on Signal Processing, Communications and Computing, Hong Kong, 5-8 August 2016, 1-6.

[45] Meng, N., Zhang, H. and Lu, H. (2016) Virtual Cell-Based Mobility Enhancement and Performance Evaluation in Ultra-Dense Networks. Wireless Communications and Networking Conference, Doha, 3-6 April 2016, 1-6. https://doi.org/10.1109/WCNC.2016.7564915

[46] Aminu, M.U., Kaleva, J. and Tolli, A. (2016) Dynamic Clustering for Max-Min Fairness with Joint Processing CoMP. International Symposium on Personal, Indoor, and Mobile Radio Communications, Valencia, 4-8 September 2016, 1-5. https://doi.org/10.1109/PIMRC.2016.7794772

[47] Papadogiannis, A., Gesbert, D. and Hardouin, E. (2008) A Dynamic Clustering Approach in Wireless Networks with Multi-Cell Cooperative Processing. IEEE International Conference on Communications, 4033-4037.

[48] Liu, J. and Wang, D. (2009) An Improved Dynamic Clustering Algorithm for Multi-User Distributed Antenna System. International Conference on Wireless Communications \& Signal Processing, Nanjing, 13-15 November 2009, 1-5.

[49] Papadogiannis, A., Gesbert, D. and Hardouin, E. (2008) A Dynamic Clustering Approach in Wireless Networks with Multi-Cell Cooperative Processing. IEEE International Conference on Communications, Beijing, 19-23 May 2008, 4033-4037. https://doi.org/10.1109/ICC.2008.757

[50] Feng, S., Feng, W., Mao, H., et al. (2015) Overlapped Clustering for Comp Transmissions in Massively Dense Wireless Networks. IEEE International Conference on Communication Systems, Macau, 19-21 November 2014, 308-312.

[51] Li, H., Tian, H., Qin, C., et al. (2012) A Novel Distributed Cluster Combination Method for CoMP in LTE-A System. International Symposium on Wireless Personal Multimedia Communications, Taipei, 24-27 September 2012, 614-618.

[52] Zhang, Z., Zhang, J. and Mu, X. (2017) Dynamic User-Centric Clustering Scheme for Uplink Multi-Cell Cooperative Systems.

[53] Liu, Z., Ma, Z. and Shi, R. (2011) Location Information Based Clustering Algorithm. Application Research of Computers, 28, 4691-4694.

[54] Zaidi, S., Hmidet, B., Affes, S., et al. (2016) User-Centric Wireless Access Virtualization Strategies for Future 5G Networks. IEEE International Conference on Ubiquitous Wireless Broadband, Nanjing, 16-19 October 2016, 1-4.

[55] Garcia, V., Zhou, Y. and Shi, J. (2014) Coordinated Multipoint Transmission in Dense Cellular Networks with User-Centric Adaptive Clustering. IEEE Transactions on Wireless Communications, 13, 4297-4308. https://doi.org/10.1109/TWC.2014.2316500

[56] Zeng, C., Ren, P., Zhang, C., et al. (2013) A Hybrid Clustering Approach in Coordinated Multi-Point Transmission System. International ICST Conference on Communications and Networking in China, Kun Ming, 8-10 August 2012, 627-631.

[57] Li, C., Zhang, J., Haenggi, M., et al. (2014) User-Centric Intercell Interference Nulling for Downlink Small Cell Networks. IEEE Transactions on Communications, 63, 1419-1431. https://doi.org/10.1109/TCOMM.2015.2402121

[58] Monserrat, J.F., Alepuz, I., Cabrejas, J., et al. (2016) Towards User-Centric Opera- 
tion in 5G Networks. Eurasip Journal on Wireless Communications \& Networking, No. 1,6 .

[59] Xu, W., Huang, J., Yang, F., et al. (2013) Dynamic Cell Clustering for CoMP in LTE-A and Its Calibrated System Level Performance Evaluation. IEEE International Symposium on Microwave, Antenna, Propagation and EMC Technologies for Wireless Communications, 71-77.

[60] Zhao, J., Zhang, T., Zeng, Z., et al. (2013) An Overlapped Clustering Scheme of Coordinated Multi-Point Transmission for LTE-A Systems. International Conference on Communication Technology, Chengdu, 9-11 November 2012, 479-484.

[61] Liu, M., Li, B. and Tong, Z. (2013) Cell Clustering and Resource Allocation Scheme for CoMP SU-MIMO. Wireless Personal Communications, 69, 1399-1411. https://doi.org/10.1007/s11277-012-0641-3

[62] Lagen, S., Agustin, A., Vidal, J., et al. (2015) Distributed User-Centric Clustering and Precoding Design for CoMP Joint Transmission. IEEE Global Communications Conference, San Diego, 6-10 December 2015, 1-7. https://doi.org/10.1109/GLOCOM.2015.7417090

[63] Zhu, X., Zeng, J., Su, X., et al. (2016) On the Virtual Cell Transmission in Ultra Dense Networks. Entropy, 18, 374. https://doi.org/10.3390/e18100374

[64] Wang, G., Duan, H. and Tan, B. (2017) Interference Coordination-Based Resource Allocation Algorithm in Ultra-Dense Networks.

[65] Sun, Y., Chang, Y., Hu, M., et al. (2015) A Cluster-Based Hybrid Access Strategy Using Non-Cooperative Game Theory for Ultra-Dense HetNet. International Conference on High Performance Computing and Communications, New York, 24-26 August 2015, 14-19. https://doi.org/10.1109/HPCC-CSS-ICESS.2015.146

[66] Hajisami, A. and Pompili, D. (2017) Dynamic Joint Processing: Achieving High Spectral Efficiency in Uplink 5G Cellular Networks. Computer Networks, 126, 44-56. https://doi.org/10.1016/j.comnet.2017.06.026

[67] Widiarti, H., Pyun, S.Y. and Cho, D.H. (2010) Interference Mitigation Based on Femtocells Grouping in Low Duty Operation. Vehicular Technology Conference-Fall, Ottawa, 6-9 September 2010, 1-5. https://doi.org/10.1109/VETECF.2010.5594576

[68] Liang, L., Wang, W., Jia, Y., et al. (2016) A Cluster-Based Energy-Efficient Resource Management Scheme for Ultra-Dense Networks. IEEE Access, 4, 6823-6832. https://doi.org/10.1109/ACCESS.2016.2614517

[69] Jiao, R., Wen, X., Lu, Z., et al. (2017) Dynamic User-Centric Clustering Algorithm Based on Energy Efficiency in Cloud-RAN. International Conference on Telecommunications, Limassol, 3-5 May 2017, 1-7.

[70] Zheng, C., Fan, J. and Luo, X. (2016) Spectrum and Energy Efficiency Analysis of Ultra Dense Network with Sleep. IEEE International Conference on Communication Software and Networks, Beijing, 4-6 June 2016, 392-396. https://doi.org/10.1109/ICCSN.2016.7586689

[71] Zhao, J., Quek, T.Q.S. and Lei, Z. (2013) Semi-Distributed Clustering Method for CoMP with Limited Backhaul Data Transfer. Wireless Communications and Networking Conference, Shanghai, 7-10 April 2013, 3776-3781. https://doi.org/10.1109/WCNC.2013.6555176

[72] Lakshmana, T.R., Tölli, A., Devassy, R., et al. (2015) Precoder Design with Limited Feedback and Backhauling for Joint Transmission. 